Results The lgNT-ProBNP in patients with UA and NSTEMI have positive correlation with their GRACE risk score, correlation coefficients were 0.40 and 0.52 , respectively $(p<0.05)$; the correlation coefficient of NT-proBNP level and GRACE risk score for all the patients $(n=126)$ was $0.59(p<0.05)$. After GRACE risk stratification, IgNT-ProBNP of high-risk group was the highest among the three groups $(p<0.05)$, however, the difference of GRACE score between middle-risk group and low-risk group had no statistical significance $(p>0.05)$. The lgNT-ProBNP in high-risk group was higher than non- high-risk group.

Conclusion Increased NT-proBNP level was associated with increased GRACE score in NSTEACS patients; NT-proBNP level of high-risk group increased significantly and was higher than nonhigh-risk group. NT-proBNP level in patients with NSTEACS was related to clinic risk and valuable for risk stratification in patients with NSTEACS.

\section{e0440 PREGNANCY-ASSOCIATED PLASMA PROTEIN-A POLYMORPHISMS AND THE RISKS OF ACUTE CORONARY SYNDROME}

doi:10.1136/hrt.2010.208967.440

${ }^{1}$ Caixia Tian, ${ }^{1}$ Weichao Qin, ${ }^{2}$ Weidong Zheng, Tangxin Liu. ${ }^{1}$ Renmin Hospital of Yunyang Medical College; ${ }^{2}$ Taihe Hospital of Yunyang Medical College

Background Pregnancy-associated plasma protein-A (PAPP-A) is known to be abundantly expressed in vulnerable plaques in arteriosclerotic disease. Studies have shown PAPP-A to be a sensitive biomarker of plaque instability and cardiovascular events in patients with acute coronary syndrome. This paper tried to determine the association of PAPP-A polymorphisms with acute coronary syndrome (ACS).

Methods A case-control study of 210 patients with ACS and 204 unrelated age and sex matched controls was performed. four single nucleotide polymorphisms (SNPs) of PAPP-A gene variants were detected by PCR-restriction fragment length polymorphism (PCRRFLP). The serum level of PAPP-A was measured using a newly developed sandwich ELISA technique based on 2 monoclonal antibodies.

Results Mean PAPP-A values were significantly higher in patients with acute coronary syndrome than in those with stable angina pectoris (29.7 vs $15.8 \mathrm{mIU} / \mathrm{l}, \mathrm{p}<0.01$ ). In samples drawn $<2 \mathrm{~h}$ after admission, the sensitivity of PAPP-A was superior $(93 \%)$ to that of CK-MB (60\%) and troponin T (61\%). In the patients with high-risk unstable angina pectoris, PAPP-A was related to the risk of nonfatal myocardial infarction $(p=0.02)$ but not death $(p=0.08)$. This result was consistent on multivariate analysis of the combination of mortality or nonfatal myocardial infarction (OR 2.65, 95\% CI 1.40 to 5.03). In patients with non-ST-elevation acute coronary syndrome and ST elevation myocardial infarctions, PAPP-A was related to the risk of death $(p=0.01)$. This was also true after adjustment for other univariate predictors of death (OR 2.19, 95\% CI 1.16 to 4.16). Multiple logistic regression analysis with risk factors such as age, male sex, smoking, hypertension, diabetes mellitus, and dyslipidemia revealed the PAPP-A IVS6+95 C allele (dbSNP: rs13290387) to be associated with an increased risk of ACS (OR, 2.44; 95\% CI 1.21 to $3.98 ; \mathrm{p}=0.018)$. The IVS6+95 (G/C) polymorphism in the PAPP-A gene has been reported 102 cases $(48.6 \%)$ were GG and 80 cases (38.1\%) were GC and 28 cases $(13.3 \%)$ were CC for the ACS group; the respective figures were 116 $(56.9 \%)$ and $70(34.3 \%)$ and $18(8.8 \%)$ in the controls. Patients carrying the $\mathrm{C}$ allele had a tendency to increased risk of ACS.

Conclusions In the early stages of non-ST-elevation acute coronary syndrome and ST elevation myocardial infarctions, PAPP-A seems to be a more sensitive marker of myocardial infarction than CK-MB and troponin T. PAPP-A seems to be valuable in predicting the outcomes of patients admitted with high-risk NSTE-ACS or STEMI PAPP-A IVS6+95 C allele is an independent risk factor for ACS even after adjustment for traditional risk factors.

\section{E0441 SAFETY OF AGGRESSIVE ANTI-THROMBOTIC THERAPY IN ELDERLY PATIENTS WITH PERSISTENT ST ELEVATED MYOCARDIAL INFARCTION UNDERWENT PRIMARY PERCUTANEOUS CORONARY INTERVENTION - A SINGLE CENTER AND SINGLE OPERATOR EXPERIENCE}

doi:10.1136/hrt.2010.208967.441

Li Yi, Hu Chengheng, Du Zhimin, Ma Hong, He Jiangui, Liu Jun. First Affiliated Hospital of Sun Yat-sen University

Objectives To evaluated the safety and efficacy of individualised anti-thrombotic therapy in elderly patients with ST-elevated myocardial infarction underwent primary PCI based on age groups. Methods Study population Between Jan. 2007 and Dec. 2008, patients with ST-elevated myocardial infarction eligible for primary PCI was assigned into 3 groups based on their ages: non-elderly group (CON, $\leq 65)$, elderly 1(ELD1, 65<age $\leq 75)$, and elderly 2 (ELD2, >75). These patients received individualised anti-thrombotic therapy based on their age group. Non-elderly patients received $300 \mathrm{mg}$ aspirin and $600 \mathrm{mg}$ clopidogrel loading dose at the emergency department and $10 \mu \mathrm{g} / \mathrm{kg}$ tirofiban loading dose were given intravenous or intra-coronary prior to intervention and followed by $0.15 \mu \mathrm{g} / \mathrm{kg} \cdot \mathrm{min}$ infusion for $36 \mathrm{~h}$. Elderly patients received $300 \mathrm{mg}$ aspirin and $300 \mathrm{mg}$ clopidogrel loading dose at the emergency department and tirofiban was given based on the thrombus burden in the culprit vessel. Clinical and angiographic parameters bleeding complications, syntax score, TIMI and CTFC coronary flow, TMP myocardial perfusion grade, in-hospital and long-term MACE, including cardiogenic death, non-fatal re-infarction, target vessel revascularization, re-hospitalisation.

Results Between Jan. 2007 and Dec. 2008, 124 patients with STelevated myocardial infarction eligible for primary PCI were enrolled. There were 48 patients in control group, 46 patients in ELD1 group, and 30 patients in ELD2 group. Patients in ELD1 group and ELD2 group had more co-morbidity factors. The complexity of coronary lesions was similar in three groups, the SYNTAX score in three groups were 17.7 $\pm 7.3,17.0 \pm 7.7$ and $16.8 \pm 6.1(p=0.829))$. The immediate angiographic outcome was also similar in three groups. The CTFC of infarction-related artery in three group were $31.4 \pm 14.1,33.3 \pm 16.9$ and $32.5 \pm 13.8(\mathrm{p}=0.279))$. TMP-3 perfusion were achieved in $79.2 \%, 71.2 \%$ and $80 \%$ patients in 3 groups. TIMI3 flow were achieved in $87.5 \%, 86.9 \%$ and $86.6 \%$ patients in 3 groups. There were no fatal bleeding and TIMI major bleeding in both groups. There was a trend of increased TIMI minor bleeding risk in ELD2 group patients.

Conclusion Our single-center and single-operator experience indicate that individualised aggressive anti-thrombotic therapy for elderly patients with ST-elevated myocardial infarction underwent primary PCI could improve myocardial perfusion and coronary flow. Individualised aggressive anti-thrombotic therapy for elderly patients with ST-elevated myocardial infarction underwent primary PCI did not increase the bleeding risk.

\section{e0442 EFFECT OF ASPIRIN AND CILOSTAZOL ON INFLAMMATORY CYTOKINES IN PATIENTS WITH ACUTE CORONARY SYNDROME}

doi:10.1136/hrt.2010.208967.442

${ }^{1}$ Hao Tang, ${ }^{2}$ Jingguo Wu, ${ }^{2}$ Qing Yang, ${ }^{3}$ Zhongfu Ma. ${ }^{1}$ Department of General Internal Medcine of The First Affiliated Hospital of Sun Yat-sen University, Guangzhou; ${ }^{2}$ Department of General Internal Medcine of The First Affiliated Hospital of Sun Yat-sen 
University; ${ }^{3}$ Department of General Internal Medcine of The First Affiliated Hospital of Sun Yat-sen University Guangzhou

Objective To investigate the effect of aspirin and cilostazol on interleukin-6 (IL-6) and high sensitive $\mathrm{C}$ reactive protein (hsCRP) and platelet-activating factor acetylhydrolase (PAF-AH) in acute coronary syndrome (ACS) patients and the difference between them.

Methods 72 patients with ACS were randomly divided into two groups: the aspirin group $(n=34)$ and cilostazol group $(n=38)$. All patients were given routine therapy including rest, oxygen inhaling, anticoagulating, reducing blood lipid levels, controlling the blood pressure. The patients in the aspirin group were given aspirin $0.1 \mathrm{~g}$ every day in addition. The patients in the cilostazol group were given cilostazol $0.1 \mathrm{~g}$ twice a day underlying the routine therapy. The course of treatment was 4 weeks. Observe the IL- 6 and hsCRP and PAF-AH in serum and on peripheral blood mononuclear cells of the patients before and after treatment.

Result The basic characteristics of the two groups were identical $(p>0.05)$. The content of IL- 6 and hsCRP were all significantly decreased after therapy $(p<0.05)$, and the content of PAF-AH were significantly increased after therapy $(\mathrm{p}<0.05)$. The content of IL-6 $(25.9 \pm 7.5$ vs $20.3 \pm 9.8 \mathrm{pg} / \mathrm{ml})$ and hsCRP $(9.5 \pm 2.1$ vs $6.1 \pm 1.9 \mathrm{~g} / \mathrm{l})$ in aspirin group (after treatment) were significantly higher than those in cilostazol group (after treatment) $(\mathrm{p}<0.05)$, and PAF-AH $\left(27.2 \pm 5.6\right.$ vs $\left.36.8 \pm 2.6 \mu \mathrm{mol} \cdot \mathrm{min}^{-1} \mathrm{l}^{-1}\right)$ in aspirin group (after treatment) were significantly lower than those in cilostazol group (after treatment) $(\mathrm{p}<0.05)$.

Conclution Aspirin and cilostazol can decrease IL- 6 and hsCRP level and increase PAF-AH level in ACS patients. And cilostazol is more effective than aspirin to inhibit the inflammatory response in ACS patients.

\section{C0443 A NEW 30-DAY MORTALITY RISK SCORE SYSTEM FOR PATIENTS HOSPITALISED WITH ACUTE MYOCARDIAL INFARCTION}

\section{doi:10.1136/hrt.2010.208967.443}

Zhao Yusheng, Xu Qiang, Wu Xingli, Xue Qiao, Gao Lei, Wang Shiwen. Institute of Geriatric Cardiology, Chinese Pla General Hospital

Objectives To derive and validate a simple scoring system that predicts risk of 30-day mortality in patients hospitalised with acute myocardial infarction (AMI).

Methods We included 5, 524 patients with AMI who hospitalised from January 1, 1993, through December 31, 2009, at Chinese PLA General Hospital in Beijing. Age, sex, comorbidity, in-hospital mortality and complications were examined for patients primarily admitted for AMI.

Results The 30-day in-hospital mortality was $9.2 \%$ in patients. Cox regression multivariate analysis showed that a history of stroke and the complications such as cardiac shock, heart failure, ventricular tachycardia/fibrillation, pneumonia, gastrointestinal bleeding, multiple organ dysfunction syndromes, being female and being older than 50 were the only independent predictors of in-hospital mortality. Using the regression coefficient as a benchmark, we calculated a convenient score. In the validation dataset, the 1,677 patients with the lowest scores had a mortality rate of $1.5 \%$ and the 1,454 patients with the highest scores had a mortality rate of $24.2 \%$.

Conclusions The study illustrates that a history of stroke, the complications, gender and age (older than 50 ) have proved to be a major prognostic marker for immediate poor outcome in the patients with AMI. The score may help to identify patients who are more likely to have a risk of in-hospital mortality within 30-days. e0444 THE VALUE OF TISSUE DOPPLER-DERIVED E/E' IN PREDICTING HEART FAILURE IN PATIENTS ADMITTED FOR UNSTABLE ANGINA AND NON ST ELEVATION MYOCARDIAL INFARCTION

doi:10.1136/hrt.2010.208967.444

Maureen Valentin, Eduardo Vicente Caguioa. Ust Hospital

Background In patients with acute myocardial infarction (AMI), diastolic function provides important prognostic information that is incremental to systolic function. Unlike other Doppler parameters of diastolic function, early mitral annulus velocity, e' appears to be relatively independent of preload. In addition, the ratio of early transmitral flow velocity, E to $e^{\prime}, \mathrm{E} / \mathrm{e}^{\prime}$ has been shown to be the most accurate predictor of left ventricle (LV) filling pressure.

Objectives The aim of this study was to determine the prognostic significance of E/e' ratio obtained by tissue Doppler imaging (TDI) among patients admitted for unstable angina (UA) and non ST elevation myocardial infarction (NSTEMI) in relation to the development of congestive heart failure (CHF)

Methods 53 patients admitted with a diagnosis of NSTEMI or UA had transthoracic echocardiogram done within $72 \mathrm{~h}$ from admission. The patients were followed up during hospital stay. The end-point was ocurrence of CHF.

Results The computed cut off value for $\mathrm{E} / \mathrm{e}^{\prime}$ ratio that would predict the development of CHF during hospital admission was 11.4. Twenty three (46\%) patients had an $\mathrm{E} / \mathrm{e}^{\prime}$ ratio $>11.4$. During hospital stay of a mean of $12.04 \pm 9.92$ days, 18 patients (34\%) had congestive heart failure. In a stepwise multivariable model, the most powerful independent prognostic indicator for the development of $\mathrm{CHF}$ was an $\mathrm{E} / \mathrm{e}^{\prime}$ ratio > 11.4 (OR 5.45 , 95\% CI 1.07 to 53.00 , $\mathrm{p}=0.050)$. The other independent predictors were history of smoking and diabetes mellitus (OR 2.69, 95\% CI 1.80 to 40.36, $\mathrm{p}=0.017$ ), use of statins (OR $0.01,95 \%$ CI 0.00 to $0.37, p=0.015$ ), $\mathrm{PV}_{\mathrm{S}}(\mathrm{OR} 0.89,95 \%$ CI 0.80 to $0.99, \mathrm{p}=0.026)$ and $\mathrm{PV}_{\mathrm{D}}(\mathrm{OR} 1.21$, 95\% CI 1.04 to $1.41, \mathrm{p}=0.013$ ).

Conclusion An E/e' $>11.4$ is a good predictor of the ocurrence of heart failure in patients with NSTEMI or UA.

\section{e0445 ADMISSION HYPOGLYCAEMIA AND HYPERGLYCAEMIA ARE ASSOCIATED WITH INCREASED MORTALITY IN OLDER PATIENTS WITH ACUTE MYOCARDIAL INFARCTION}

doi:10.1136/hrt.2010.208967.445

${ }^{1}$ Yang Shiwei, 'Zhou Yujie, ${ }^{2} \mathrm{Hu}$ Dayi, ${ }^{1}$ Nie Xiaomin, ${ }^{1}$ Liu Yuyang, ${ }^{1}$ Shi Dongmei, ${ }^{1}$ Guo Yonghe. ${ }^{1}$ Beijing Anzhen Hospital Affiliated To Capital Medical University; ${ }^{2}$ People's Hospital Affiliated To Peking University

Objective The aim was to assess the association between fasting plasma glucose (FPG) levels on admission and mortality in older patients with acute myocardial infarction (AMI), and compare the effects of FPG levels on outcomes in the context of contemporary treatments, including medical therapy, percutaneous coronary intervention and coronary artery bypass grafting.

Methods From April 2004 to October 2006, 1854 older (age $\geq 65$ years) AMI patients were enrolled in the Beijing Elderly Acute Myocardial Infarction Study (BEAMIS) consecutively. Patients were categorised into 4 groups: hypoglycemia group ( $N=443,23.9 \%)$, $\mathrm{FPG} \leq 5 \mathrm{mmol} / \mathrm{l}$; euglycemia group ( $\mathrm{N}=812,43.8 \%), 5.1 \mathrm{mmol} / \mathrm{l} \leq$ FPG $\leq 7 \mathrm{mmol} / 1$ (5-7 mmol/l); mild hyperglycemia group $(\mathrm{N}=308$, $16.6 \%), \quad 7.1 \mathrm{mmol} / \mathrm{l} \leq \mathrm{FPG} \leq 9 \mathrm{mmol} / \mathrm{l} \quad(7-9 \mathrm{mmol} / \mathrm{l}) ;$ and severe hyperglycemia group ( $\mathrm{N}=291,15.7 \%), \quad \mathrm{FPG} \geq 9.1 \mathrm{mmol} / \mathrm{l}$. The primary end point was in-hospital and 3-year all-cause mortality from the day of admission. 